\title{
LA INVESTICACIÓN CIENTÍFICA DE LA BIODIVERSIDAD COMO INGREDIENTE INDISPENSABLE DEL ECOTURISMO
}

\section{E. Galante Marcos}

Instituto Nacional DE Biodiversidad, INBio (COSTA Rica)

\section{INTRODUCCIÓN}

De la fusión del turismo de naturaleza y el turismo sostenible, surge lo que conocemos como Ecoturismo: modalidad del turismo de naturaleza realizado de una forma sostenible. Según este principio, no todas las actividades turísticas en la naturaleza son necesariamente "eco", pero sí todas las actividades ecoturísticas se centran en la naturaleza.

El ecoturismo es un turismo responsable con el ambiente y las comunidades locales que involucra esta actividad, y aprovecha de forma sostenible la biodiversidad, obteniendo así beneficios económicos.

Para que una actividad o un tipo de turismo pueda ser considerado ecoturismo, ha de cumplir también otros requisitos:

- En el ámbito social y cultural debe fortalecer la comunidad en la que está inmerso, respetar sus costumbres y propiciar el empleo de miembros de la comunidad.

- En cuanto al ámbito económico, debe tener un diseño y una escala de operación a la medida de las posibilidades y características del lugar.
- Y en el ámbito ambiental, debe apoyar activamente la conservación, ofrecer a los clientes el contacto directo con la naturaleza, haciendo uso de buenas prácticas ambientales, y ofrecer información e interpretación ambiental a los usuarios de ese servicio.

La Estrategia Global para la Biodiversidad se refiere a la trilogía de Conocer, Salvar, y Usar. Un círculo indivisible que se basa en la idea de que conociendo la biodiversidad podremos salvarla y determinar los beneficios sociales y económicos que nos puede aportar haciendo un uso sostenible de ella, lo cual promoverá su conservación a largo plazo (Figura 1).

Esta estrategia está directamente relacionada con la filosofía y el porqué del ecoturismo, viéndolo como un uso sostenible que se obtiene de la biodiversidad, que promueve su conservación y que tiene un gran pilar base, que es el estudio, conocimiento e investigación de esa biodiversidad de la que se beneficia y a la cual conserva.

La investigación científica es muchas veces una actividad en la sombra, desconocida y no siempre 


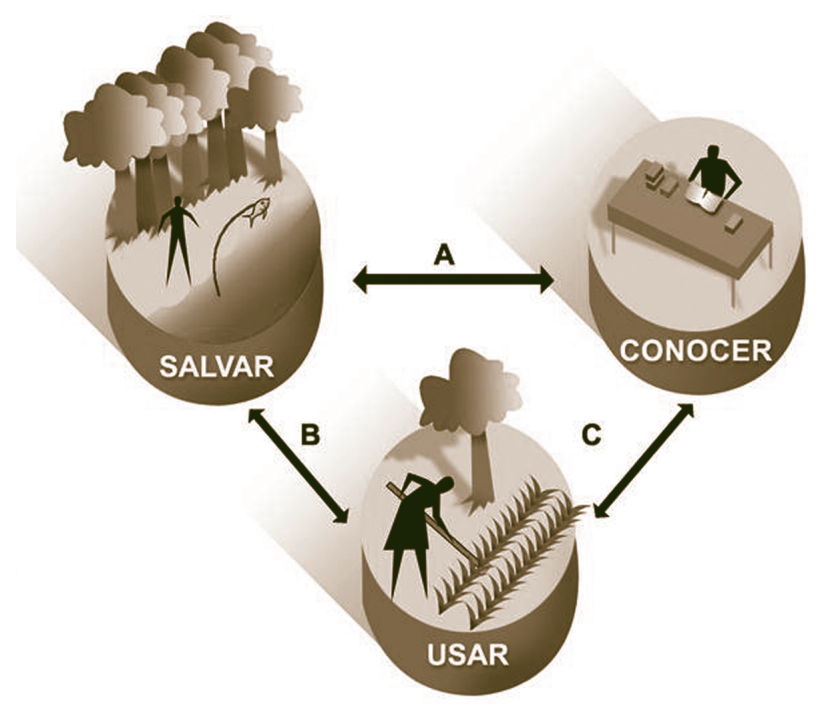

Figura 1. Trilogía Conocer, Salvar, y Usar (Estrategia global para la conservación de la biodiversidad).

valorada, entre otras cosas, por no ser entendida por la gran mayoría de las personas. El ecoturismo es una muestra directa de la aplicabilidad de tales investigaciones y de la importancia de las mismas, ya que son la base de todas y cada una de las actividades que el ecoturismo engloba, y es a su vez una forma de comunicación de la ciencia.

\section{EL PAPEL DE LA INVESTIGACIÓN CIEN- TÍFICA EN EL ECOTURISMO}

Desde un punto de vista estrictamente turístico, cuando se habla de Producto significa la suma de atractivos (naturales, históricos y culturales), infraestructura (hoteles, senderos...), equipamiento (lanchas, binoculares...), actividades (observación de animales, caminatas por el bosque...) y servicios (alimentación, limpieza, guías, interpretación ambiental...).

Por una parte, en la primera etapa de identificación o diseño del producto es necesario identificar los atractivos naturales, y el hecho de contar con un aporte científico permite la elaboración de inventarios de flora y fauna para conocer la riqueza natural del sitio, así como para la valoración de la "materia prima" con la que se cuenta en la potencial actividad turística.

Las actividades del ecoturismo se fundamentan en la naturaleza y para llevarlas a cabo es preciso el conocimiento "del terreno de trabajo". Algunas especies de aves, como el quetzal, atraen a cientos de turistas a los bosques nubosos de Costa Rica, sin embargo la visita al bosque no garantiza la aparición del ave. Es necesario, en primer lugar por supuesto, conocer que esta especie vive en dicho ecosistema, por otra parte, conocer los árboles en los cuáles anida y las épocas de reproducción, los frutos de los que se alimenta y la altitud a la que se encuentran, para poder seguir su rastro de una forma más certera. El conocimiento del ecosistema en el cual habita, el registro de sus costumbres y comportamientos y el estudio a fondo de la especie, hace más fácil su localización y la consecuente satisfacción de los turistas, lo que beneficiará directamente al territorio donde se da el evento natural.

Las anidaciones de tortugas marinas o las migraciones de grandes cetáceos, son otros ejemplos de eventos de la naturaleza capaces de movilizar grandes cantidades de turistas, que deseosos de disfrutar de una experiencia natural, viajan a otros países.

Para conocer estos eventos y sus porqués es imprescindible la investigación científica sobre la biodiversidad, gracias a la cual podríamos elaborar calendarios de eventos naturales a lo largo del tiempo y del espacio. Pero esta investigación es necesaria también, para conocer el impacto de las actividades que llevamos a cabo en la naturaleza y establecer normativas y formas de llevarlas a cabo, respetando los ciclos naturales, con el fin de que sea una actividad sostenible a largo plazo y merezca el nombre de ecoturismo.

Por otra parte, la investigación también permite, que a partir de las especies identificadas, se disponga de información específica de cada una de ellas, de su historia natural, sus características y peculiaridades. Dicha información se utilizará como un servicio complementario, aplicándola de diferentes formas: a través de caminatas guiadas, interpretación ambiental, guías de campo... y toda una larga lista de servicios destinados a los clientes consumidores del producto turístico (Figura 2).

Estos servicios que forman parte del ecoturismo, lo convierten en una forma de comunicación de los fenómenos de la biodiversidad y del conocimiento generado a cerca de los mismos. Significa la transmisión de esa información a los turistas, lo que se conoce como interpretación ambiental, y esa transmisión no es posible sin un paso previo de generación del 
conocimiento. Una caminata en el bosque puede ser un bonito paseo en la naturaleza entretenido y saludable, o puede ser también algo similar a leer un libro de historias fascinantes, de detalles y porqués y hasta de cuentos para niños. Cuando un paseo por el campo se convierte en un descubrimiento y una traducción de la naturaleza, eso es ecoturismo y para escribir dichas historias, ha sido necesaria la investigación de los componentes de ese bosque.

En Costa Rica, en los años noventa, con el auge del ecoturismo, aquellos que aspiraban a ejercer de guías de turismo, sabían que había dos lecturas obligatorias: el libro Costa Rican Natural History, de JANZEN (1983), quien compiló numerosos artículos de diversos investigadores sobre la historia natural del territorio costarricense y de algunas especies seleccionadas, y la Guía de aves de Costa Rica, de STILES \& SKUTCH (1995), con ilustraciones de las aves costarricenses, información de su hábitat, historia natural etc... que originó el desplazamiento de numerosos ornitólogos y amantes de las aves a raíz de su publicación y de la difusión de esa información. Estas dos publicaciones estaban dirigidas a la comunidad científica internacional y otros interesados en los procesos naturales, entre los que no se preveía el sector turismo, que fue un público inesperado de un sector emergente y en formación, sediento de información y recursos para crecer y expandirse.

Gracias a estas y otras investigaciones y publicaciones, un árbol con espinas (Acacia collinsii Saff.) y hormigas (Pseudomyrmex ferruginea Smith), pasa a ser una interesante relación simbiótica, en la que las hormigas se refugian dentro de las espinas huecas del árbol y se alimentan del néctar que éste les ofrece, obteniendo así protección y alimento, a cambio de los trabajos de limpieza del tronco y de la defensa frente a los herbívoros. Del pequeño mundo de los insectos pasamos a las plantas epifitas, algunas de las cuales son familia de la piña, las bromelias que sirven de refugio a muchos animales (Figura 3), como las larvas de unos dípteros llamados sírfidos, los cuales de adultos se asemejan a las abejas y avispas para evitar así ser comidos por los depredadores. Entre las hojas de las bromelias, también se pueden encontrar los renacuajos de unas pequeñas ranas tóxicas de color rojo (Dendrobates pumilio Schmidt), que depositan sus huevos en el agua de la lluvia acumulada en las hojas de estas plantas. Estas pequeñas ranas, aunque tóxicas, son uno de los alimentos del guardabarranco (Momotus momota (Linnaeus, 1760)), una hermosa ave verde y azul, con una larga cola, a la que llaman así porque construye sus nidos en los barrancos y desniveles.

Por otra par-

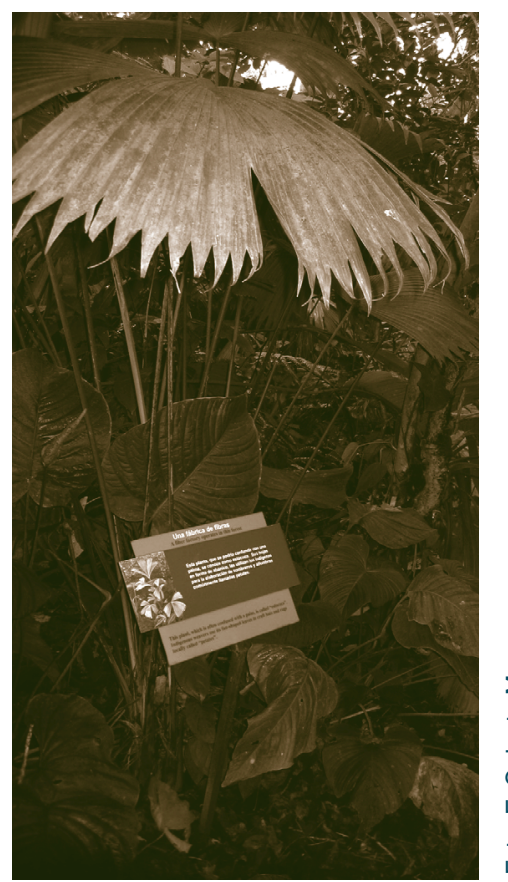

Figura 2. Rótulo de interpretación ambiental. te, con las investigaciones científicas y el estudio del medio, se abre un campo inexplorado del que pueden originarse multitud de productos nuevos que están aún por inventar. Recordemos por ejemplo, que las tirolinas conocidas como Canopy en muchos países eran plataformas que utilizaban los científicos para investigar el dosel del bosque y la flora y fauna allá existentes. En la actualidad, el Canopy es un producto del turismo directamente ligado a la naturaleza, que permite a los turistas disfrutar del bosque desde un punto de vista diferente, convirtiéndolos en aves por cortos periodo de tiempo mientras se deslizan por cables de árbol en árbol (Figura 4).

Estas y otras cientos de historias más, son parte del conocimiento generado por el sector científico, aplicado y divulgado por el sector turismo.

Las publicaciones científicas sobre la biodiversidad les sirven a los usuarios directos de esa biodiversidad, guías de turismo dueños de hoteles etc..., como información de transmisión a los turistas, convirtiéndolos así en cómplices de la ciencia y en los mayores defensores de la biodiversidad, por tener la oportunidad de conocerla y obtener de ella beneficios directos. 


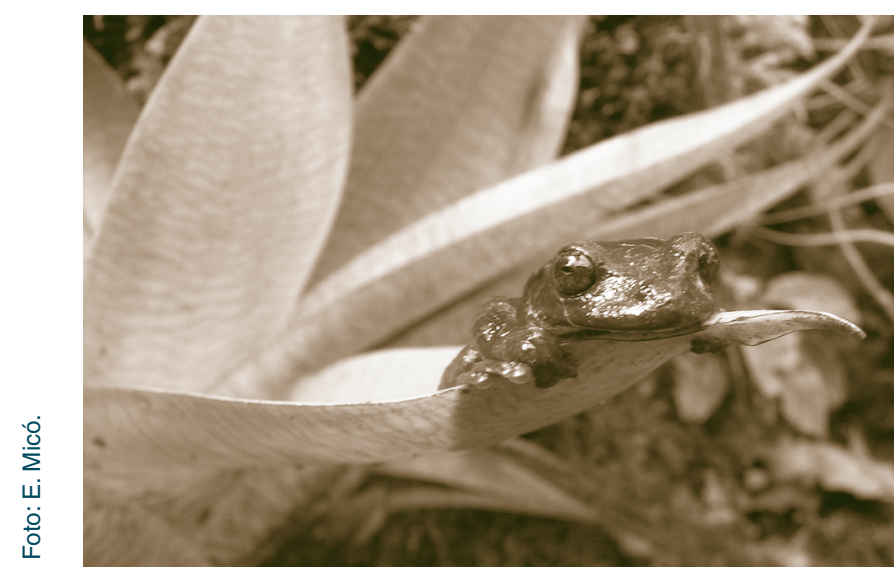

Figura 3. Detalle de una Bromelia albergando una rana en su interior.

Además, estas y otras historias, sorprenden y despiertan la curiosidad de los ecoturistas durante sus días de viaje a lo largo de los diferentes ecosistemas del mundo. A través de estas historias las investigaciones científicas, dejan de ser desconocidas para ser aplicadas directamente a una actividad de recreo y disfrute como es el turismo, sirviendo así como entrada al mundo maravilloso de la naturaleza. Estas investigaciones en boca de guías expertos, y transmitidas a través de la interpretación ambiental, suponen una traducción sencilla y amena de los fenómenos naturales y convierten una caminata $\mathrm{u}$ otra actividad en la naturaleza, en más que una forma de entretenimiento, pasa a ser una actividad recreativa de educación ambiental del turista.

Si bien se ha puesto de relieve la importancia de la investigación científica sobre la biodiversidad, como generadora de información para ser transmitida y aplicada directamente en las actividades de ecoturismo, es necesario también destacar su importancia como punto de partida para la elaboración de nuevos productos de la naturaleza que complementan la oferta ecoturística ya existente. Los mariposarios, serpentarios y acuarios, son ejemplos de productos en los que la investigación inicial es imprescindible, ya que es necesario conocer el hábitat, la alimentación, el ciclo de vida etc.... de las especies para poder exhibirlas con éxito en cautiverio propiciando su reproducción con el fin de obtener exhibiciones sostenibles.

Por otra parte, la investigación y divulgación de ese conocimiento sobre biodiversidad, es un meca- nismo excelente para la identificación de nuevos productos potenciales para diferenciar la oferta turística de un país o región, fortaleciendo así la competitividad y ofreciendo la posibilidad de posicionarse como país o región multidestino.

Como ejemplo de todo lo expuesto está $\mathrm{La}$ Reserva Privada Monteverde, en Costa Rica. A principios de los 70 un investigador de aves enamorado de Monteverde y de su enorme riqueza natural, entabló relaciones con los habitantes de esa zona, una comunidad cuáquera, para establecer el área como reserva natural. A través del Centro Científico Tropical y unos fondos que esta ONG tenía para el establecimiento de reservas privadas con fines científicos y educativos, se consiguió crear la Reserva Privada Monteverde administrada por dicha ONG y la cual se fue expandiendo al recibir donaciones de otras instituciones y de científicos amantes de las aves. Desde ese momento, se convirtió en un destino de científicos y naturalistas.

Años más tarde una cadena de televisión se interesó en hacer un reportaje sobre la reserva. A partir de la transmisión de dicho documental, las visitas de personas ajenas a la ciencia, pero con un profundo respeto e interés por la naturaleza, comenzó a crecer y se fue desarrollando el turismo alrededor de la actividad científica. En la actualidad Monteverde se ha convertido en uno de los principales destinos de Costa Rica, con una amplia oferta de turismo en la naturaleza y de ecoturismo. Las actividades turísticas que se llevan a cabo en una parte de la reserva, permiten subvencionar los gastos administrativos y de vigilancia, y las actividades de educación ambiental con diferentes públicos de la reserva, que alcanza las 5.000 hectáreas de bosque tropical nuboso.

Monteverde es un ejemplo de cómo a partir de la investigación científica se genera la actividad turística, y cómo la información generada tiene una aplicación directa.

Tal es la importancia que se le ha llegado a dar a la relación ciencia-turismo, que el ciclo de conferencias con el que se inaugura la feria de turismo de Costa Rica, tuvo como principales ponentes en el 2005, a miembros de las instituciones más destacadas a nivel nacional por su investigación científica. Los representantes de las diferentes instituciones, expusieron los esfuerzos realizados en investigación 


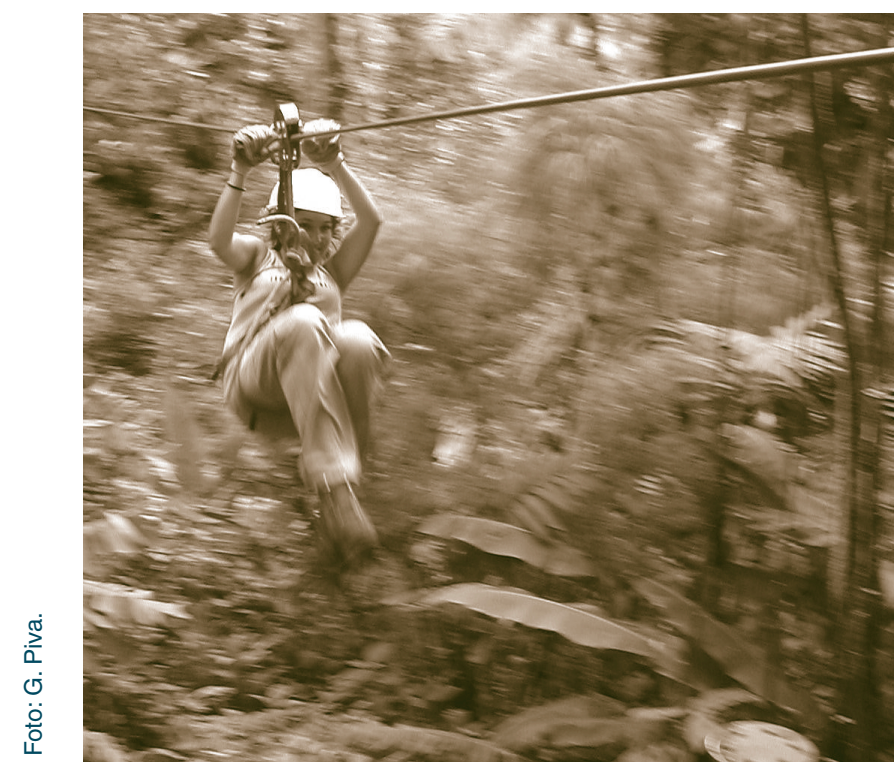

Figura 4. Tirolina conocida como "canopy".

de la biodiversidad costarricense y en la divulgación de esta información de diferentes formas. Los destinatarios de estas charlas, fueron los diferentes gremios del turismo, todos interesados en incorporar el componente científico y los conocimientos resultantes en la creación de nuevos productos o en la renovación y mejora de los ya existentes.

\section{¿CÓMO SE FUNDEN EN LA ACTUALI- DAD LA CIENCIA Y EL ECOTURISMO?}

En la actualidad, la ciencia se incorpora en el producto ecoturístico de diferentes formas. La forma más directa es a través de la capacitación en materia de biodiversidad e interpretación ambiental a los guías naturalistas, con el fin de que sean los transmisores de los fenómenos naturales del lugar. Otras modalidades que se están dando actualmente para la comunicación de la naturaleza en Costa Rica son la redacción de guías de campo, láminas de campo con imágenes y nombres de animales, hongos, plantas..., interpretación de senderos, mariposarios, ranarios..., diseño de exhibiciones interactivas, charlas y caminatas en el campo con finalidad científica. La incorporación de actividades como recolectas de hongos comestibles, colocación de trampas de luz por la noche para la observación de insectos nocturnos, pequeñas capacitaciones participativas en las que el turista aprende a identificar ranas, plantas y otros animales, convierte al turista en investigador o explorador durante un día.

Esta nueva tendencia se genera dentro del ecoturismo, pero surge como una forma más educativa de entender el turismo. Podría ser una forma de ecoturismo educativo, si fuera necesaria la invención de un nuevo nombre. Es una actividad recreativa y turística, cuya área de interés es la naturaleza, sus procesos, sus mecanismos y sus formas de estudiarla. De esta forma ciencia y sociedad se unen a través del turismo, transmitiendo de fondo un mensaje de conservación, ya que da a conocer la naturaleza en su estado más puro. Si es verdad el dicho que reza que "conservamos sólo lo que amamos, amamos sólo lo que entendemos y entendemos sólo aquello que nos ha sido enseñado", esta modalidad de turismo y transmisión de la ciencia, parece ser una de las formas de conservación y educación ambiental posibles.

\section{CONCLUSIONES}

Los ejemplos de la relación ciencia y turismo no están del todo sistematizados ni registrados, por lo que aunque es una conexión lógica, el campo del ecoturismo no le ha dado a la ciencia el reconocimiento que merece como herramienta de generación del conocimiento para llevar a cabo un uso sostenible de la biodiversidad y promover así su conservación.

La ciencia se pone al servicio de la sociedad y se transforma en una herramienta de trabajo para muchas personas que poco a poco profesionalizan su trabajo de guías de turismo y comunicadores de los fenómenos naturales.

Es necesaria, la comunicación por parte de los científicos de sus investigaciones y la formación de expertos capaces de adaptar esa información técnica y especializada en información cercana al público en general. Es necesario por tanto el compartir la información científica con la sociedad.

Cuanto más se conozca la biodiversidad de un país o región, tantas más posibilidades habrá de diseñar diferentes productos y de multiplicar la oferta. 